\title{
Spatially Periodic Electromagnetic Force Field For Plasma Confinement and Control
}

\author{
C.A. Ordonez ${ }^{*}$, J.L. Pacheco, D.L. Weathers
}

Department of Physics, University of North Texas, Denton, Texas 76203, USA

\begin{abstract}
A scientific concept referred to as a "force field" is defined as a short-range, static electromagnetic field that can reflect a charged particle of either sign of charge that approaches at any angle of incidence. A force field is envisioned as consisting of a spatially periodic sequence of magnetic cusps that are electrostatically plugged using applied electrostatic voltage variations similar to those found in nested Penning traps. The effective range of the force field would be small compared to the dimensions of a nearby source of charged particles, such as a plasma confined by the force field. A theoretical understanding is developed of the single-particle reflection properties of a force field, considering the incident charged particles to have a non-drifting, isotropic velocity distribution. Classical trajectory Monte Carlo simulations and analytical modeling are employed. The initiation of an experimental effort to study force fields is described.
\end{abstract}

Keywords: Plasma, confinement, spatially periodic, force field.

\section{INTRODUCTION}

A scientific concept referred to here as a "force field" was found in searching for an alternative to existing plasma confinement and control methods that are used for antihydrogen-related research. A force field is defined here as a short-range electromagnetic field that is designed to reflect a charged particle of either sign of charge that approaches at any angle of incidence. An existing plasma confinement approach, the Penning trap, produces an electric potential well along a magnetic field to confine a non-neutral plasma [1]. A nested Penning trap produces oppositely signed nested electric potential wells along a magnetic field to confine oppositely signed plasma species [2-7]. Fig. (1) illustrates the spatial dependence of an electric potential that forms nested electric potential wells. Nested Penning traps have been used to mix antiprotons and positrons such that antihydrogen is produced [8-12] and trapped [11, 12]. An ultimate goal of the antihydrogen research is to conduct experimental tests of CPT (charge conjugation, parity, and time reversal) and gravity symmetries [13-19].

It is not clear whether nested Penning traps can produce antihydrogen atoms with sufficiently low energies and also in sufficient numbers for conducting high precision CPT and gravity measurements, because there exist numerous conflicting issues [3,20]. Some of the issues are being addressed, as indicated by recent advances [12, 21-24]. An ideal plasma confinement approach for antihydrogen studies would be capable of providing long confinement times for a cold, dense non-drifting (e.g., non-rotating) plasma of any desired size. Force fields might ultimately provide such confinement.

*Address correspondence to this author at the Department of Physics, University of North Texas, Denton, Texas 76203, USA; Tel: (940)5652626; Fax: (940)5652515; E-mail: cao@unt.edu

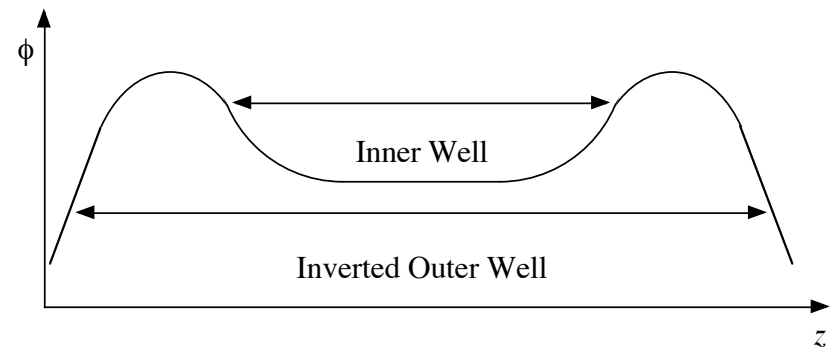

Fig. (1). Electric potential produced parallel to a magnetic field within a nested Penning trap that, under certain conditions, can confine two oppositely signed plasma species with overlapping confinement volumes. A plasma species comprising positively charged particles would be confined within the inner well. A plasma species comprising negatively charged particles would be confined within the outer well.

A force field would be produced by an artificially structured boundary that generates a spatially periodic static electromagnetic field, which has a period and an effective range that are much smaller than the dimensions of a plasma that may be confined by the field [25-29]. Fig. (2) illustrates a simple wire configuration for producing a spatially periodic magnetic field. Suppose that an unmagnetized onecomponent Maxwellian plasma drifts in the $x$ direction above the artificially structured boundary illustrated in Fig. (2). The average value of $\theta_{0}$, which has a value in the range $\pi / 2<\theta_{0} \leq \pi$ for charged particles that travel toward the artificially structured boundary, depends on the relative values of the drift speed and the charged-particle thermal speed. There can exist an effective limit on $\theta_{0}$ for particles to be reflected by the field. If the effective limit on $\theta_{0}$ is less than $\pi$, which is the case for the artificially structured boundaries studied in Refs. [25-29], then the plasma must drift faster than the charged-particle thermal speed so that the 
vast majority of incident charged particles will be reflected by the field.

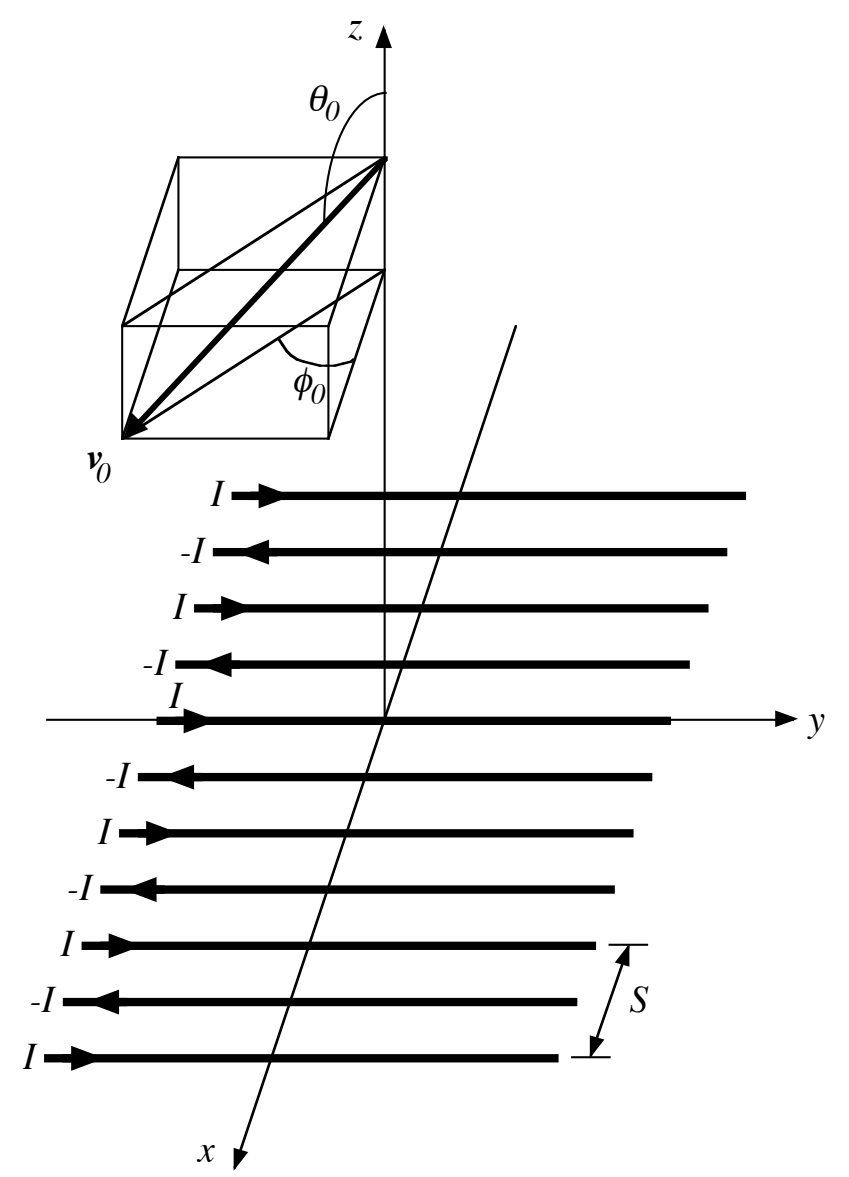

Fig. (2). The wire configuration of a simple artificially structured boundary that would produce a spatially periodic magnetic field if the wires carry currents in alternating directions. The vector $\mathbf{v}_{0}$ represents the initial velocity of a charged particle that travels toward the artificially structured boundary. The angles $\theta_{0}$ and $\varphi_{0}$ are used to describe the direction of $\mathbf{v}_{0}$.

A force field as defined here would reflect a charged particle of either sign of charge that approaches at any angle of incidence. Fig. (3) illustrates a possible artificially structured boundary for producing a force field. Fig. (3) also illustrates a configuration in which a force field may be used to confine a plasma. The force field consists of a spatially periodic sequence of magnetic cusps that are electrostatically plugged. A comprehensive review of research related to electrostatic plugging of magnetic cusps is provided in Ref. [30]. More recent research that is directly or indirectly associated with electrostatic plugging of magnetic cusps includes antihydrogen-related research [10, 31-33] and fusion-related research [34]. It should also be noted that recent research has been reported involving plasmas confined or controlled by multiple magnetic cusps $[35,36]$. The electrostatic-plugging research described in Refs. [30, 32] employed an applied electric field for reflecting electrons (or positrons), with ion (or antiproton) confinement enhancement considered to occur as a result of a buildup of negative (or positive) space charge. For the concept illustrated in Fig. (3), an electrostatic voltage variation similar to that found in nested Penning traps would be applied for providing simultaneous electrostatic plugging for oppositely signed charged particles. The concept does not rely on the establishment of a confinement equilibrium in which one species is electrically confined using the space charge of another species, although such an equilibrium is not precluded from being used with the concept.

The charged particle trap illustrated in Fig. (3d) consists of a sequence of electrostatically plugged coaxial ring cusps. The diameters of the ring cusps vary axially, with the smallest rings located near the axial ends of the confinement volume. Two point cusps that are coaxial with the ring cusps are located at the axial ends of the confinement volume.

In Sec. 2, a theoretical model of the magnetic field of a force field is developed. The theoretical model describes a sequence of noncurved line cusps. (A noncurved line cusp is produced, for example, between the straight sections of two adjacent D-shaped magnetic coils with oppositely directed currents.) Each cusp has a region within which the magnetic field has approximately straight magnetic field lines. The magnetic field is uniform within a region of straight field lines for noncurved line cusps and point cusps, but is only approximately uniform for ring cusps of sufficiently large radius. In Sec. 3, a classical trajectory Monte Carlo (CTMC) simulation is developed for charged particles that travel into the magnetic field. The particles are assumed to originate from a source with a monoenergetic isotropic velocity distribution. The fraction of particles that enter the cusps and reach the straight field-line regions is evaluated and fit with a function. The particle density profile within the straight field-line regions is also evaluated and fit for particles traveling into the cusps. The fits are used in Sec. 4 to evaluate the fraction and density profile of charged particles having a non-drifting isotropic Maxwellian velocity distribution that enter the cusps and reach the straight fieldline regions. Because the underlying analytical model on which the CTMC simulation is based is written in terms of dimensionless parameters, the results obtained in Sec. 4 apply for both ions and electrons. In Sec. 5, the relevance of plasma confinement within nested Penning traps to the electrostatic plugging employed by a force field is discussed. In Sec. 6, the initiation of an experimental effort to study force fields is described. Concluding remarks are in Sec. 7.

\section{THEORETICAL MODEL OF THE MAGNETIC FIELD}

As illustrated in Fig. (2), a spatially periodic magnetic field can be produced by a planar sequence of parallel wires with alternating currents. Define a Cartesian coordinate system with coordinates $(x, y, z)$ and unit vectors $(\hat{\mathbf{i}}, \hat{\mathbf{j}}, \hat{\mathbf{k}})$, such that the planar sequence is located at the $z=0$ plane, each wire is parallel to the $y$ axis, and the wires are evenly spaced with a separation $S / 2$ between adjacent wires. The spatial period of the magnetic field produced by such a planar sequence of wires is $S$, provided adjacent wires carry oppositely directed currents of equal magnitude. For wires of infinitesimal width, the magnetic field produced by such a planar sequence of wires is given by [26]

$\mathbf{B}_{1}(x, y, z)=2 \mu_{0} I_{1} S^{-1} \beta_{1}\left(z S^{-1}, x S^{-1}\right)$, 

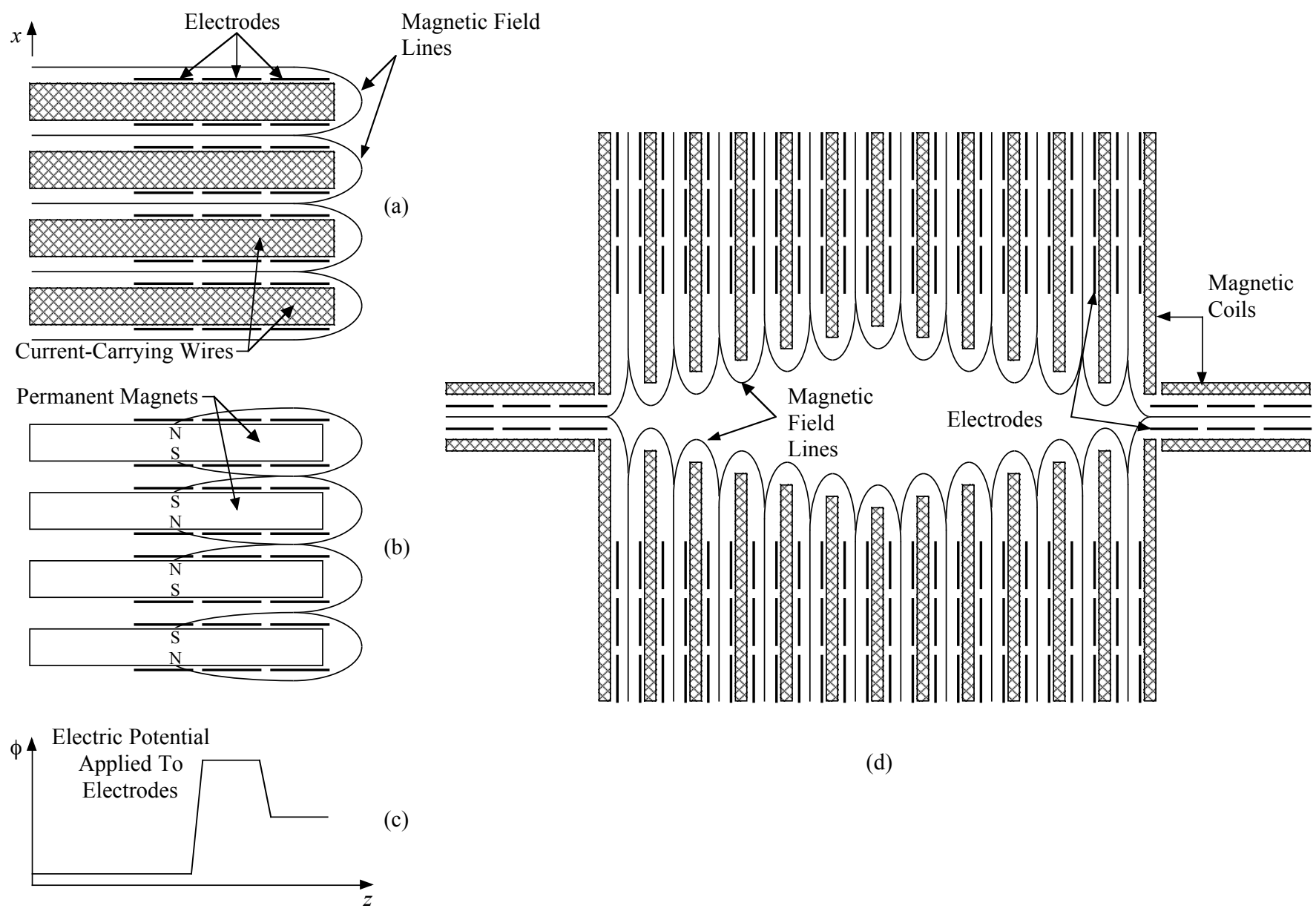

(d)

Fig. (3). Illustrations of a few spatial periods of (a) the electrode and current-carrying wire configuration and (b) permanent magnet configuration of an artificially structured boundary that produces a force field, and (c) the electric voltage applied to the electrodes. Also shown is (d) an illustration of an axisymmetric charged particle trap based on the force field concept. The applied electric voltage in (c) is similar to that applied to one side of a nested Penning trap. For the illustrations shown in (a) and (b), charged particles would be incident from the right.

where

$\beta_{1}\left(z_{n}, x_{n}\right)=\sin \left(2 \pi x_{n}\right) \cosh \left(2 \pi z_{n}\right)\left[\cos \left(4 \pi x_{n}\right)-\cosh \left(4 \pi z_{n}\right)\right]^{-1} \hat{\mathbf{k}}$

$-\cos \left(2 \pi x_{n}\right) \sinh \left(2 \pi z_{n}\right)\left[\cos \left(4 \pi x_{n}\right)-\cosh \left(4 \pi z_{n}\right)\right]^{-1} \hat{\mathbf{i}}$,

$\left|I_{1}\right|$ is the current magnitude of any one wire, $\mu_{0}$ is the permeability of free space, and SI units are used. Here, $\left(x_{n}, y_{n}, z_{n}\right)$ are normalized Cartesian coordinates with $x_{n}=x / S, y_{n}=y / S$, and $z_{n}=z / S$. A wire located at $x=0$ and $z=0$ carries a current $I_{1}$, which is positive (negative) if the current is directed in the positive (negative) $\hat{\mathbf{j}}$ direction.

For the present study, multiple planar sequences of parallel wires, which are illustrated in Fig. (4), are considered. The magnetic field produced by the configuration is given by

$\mathbf{B}(x, y, z)=B_{m} \beta\left(z S^{-1}, x S^{-1}\right)$,

where $\beta\left(z_{n}, x_{n}\right)=\sum_{i=1}^{N} \eta_{i} \beta_{1}\left[z_{n}+(i-1) \Delta z_{n}, x_{n}\right]$

Here, $B_{m}=2 \mu_{0} I_{0} / S ; \quad N$ is the number of planar sequences of wires, which are separated by a normalized distance $\Delta z_{n}=\Delta z / S ; \eta_{i}=I_{i} / I_{0}$ represents dimensionless currents, where $I_{i}$ is the current of a wire located at $x=0$ and $z=-(i-1) \Delta z$; and $I_{0}$ has a positive value and is introduced so that $\beta$ is dimensionless. It can be shown that $\nabla \cdot \mathbf{B}=0$ is satisfied. The amplitude of the magnetic field ripple can be kept below a desired maximum value by choosing a sufficiently small value for $\Delta z_{n}$. The range in $z_{n}$ values over which the magnetic field ripple can be kept below a desired maximum value depends on the value of $N$ and on the relative values of $\eta_{i}$. Fig. (5) shows the magnetic field produced with $N=10, \Delta z_{n}=0.25, \eta_{1}=\eta_{10}=1.27$, and $\eta_{i}=1$ for $2 \leq i \leq 9$. With these values, the ripple in the magnetic field strength is less than $2 \%$ midway between any 


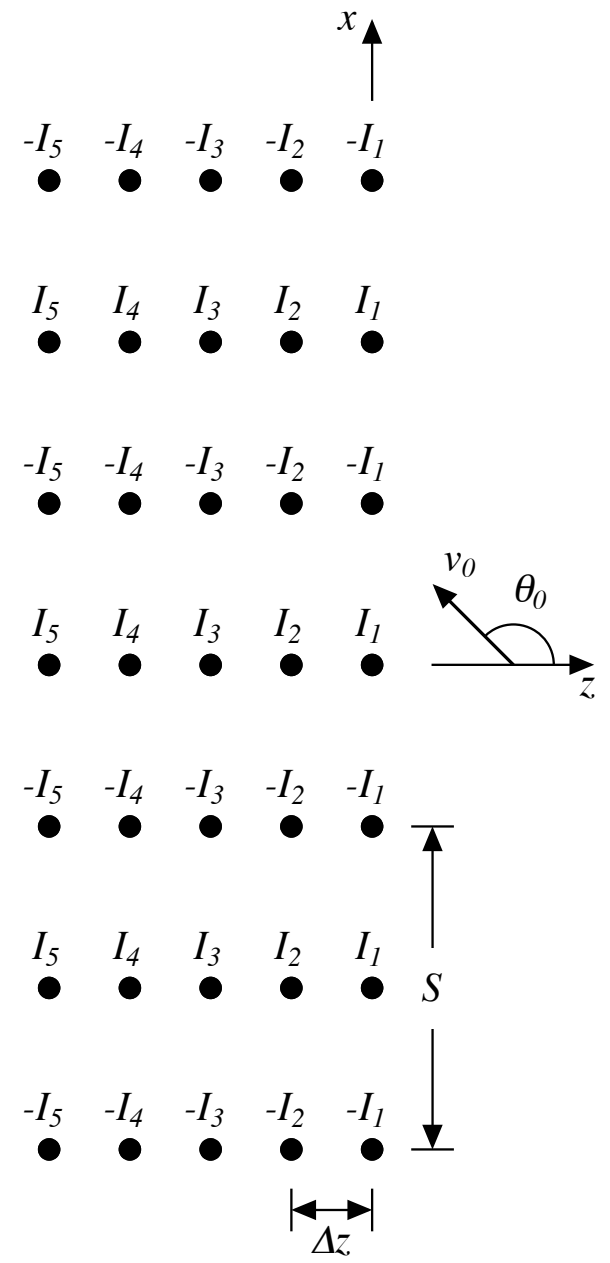

Fig. (4). Illustration of five planar sequences of current-carrying wires. Also shown are some coordinates of a configuration-space Cartesian coordinate system $(x, y, z)$ and of a velocity-space spherical coordinate system $\left(v_{0}, \theta_{0}, \varphi_{0}\right)$. (The $y$ and $\varphi_{0}$ coordinates are not shown.)

two horizontal rows of wires [i.e., at $x_{n}=0.25(1+2 j)$, where $j$ is an integer] within the range $-2.07 \leq z_{n} \leq-0.18$. The magnetic field strength decreases rapidly over a few spatial periods from the $z_{n}=0$ plane in the $\hat{\mathbf{k}}$ direction. For example, the field magnitude normalized by $B_{m}$ is calculated to have the following values: $\beta\left(z_{n}=5, x_{n}=0\right)=3.5 \times 10^{-14}$ and $\beta\left(z_{n}=0.1, x_{n}=0\right)=1.1$.

\section{CLASSICAL TRAJECTORY MONTE CARLO SIMULATION}

A CTMC simulation has been developed for charged particles that travel into the magnetic field described by Eq. (3). Each parameter used for a simulation is normalized such that it is dimensionless. The same symbol is used for normalized and un-normalized parameters that correspond to one another, except that normalized parameters have a subscript $n$ attached to them. The quantities used for normalization are the spatial period $S$ of the magnetic field and a particle's mass $m$, charge $q$, and kinetic energy $K$.
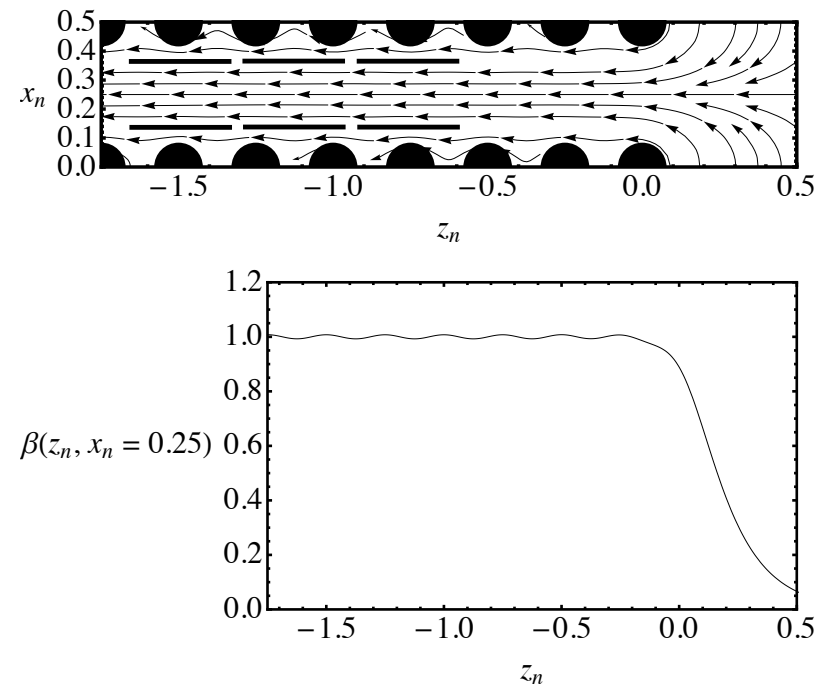

(b)

Fig. (5). Magnetic field produced by ten planar sequences of current-carrying wires $(N=10)$, with $\Delta z_{n}=0.25, \eta_{1}=\eta_{10}=1.27$, and $\eta_{i}=1$ for $2 \leq i \leq 9$. Shown are (a) the vector field between two rows of wires and (b) the field magnitude normalized to $B_{m}$ midway between two rows of wires. The solid semicircles in (a) indicate wire locations. Also shown in (a) are six solid straight lines that represent possible locations of six electrodes, which could be used to produce an electric field similar to that produced by one side of a nested Penning trap.

The kinetic energy $K$ is conserved if only a magnetic force is present. The normalized values of the quantities used for normalization are unity, $m_{n}=q_{n}=K_{n}=S_{n}=1$. Various other normalized parameters are written in terms of unnormalized parameters as follows: The particle's normalized position, velocity, and acceleration are $\mathbf{x}_{n}=\mathbf{x} / S$, $\mathbf{v}_{n}=\mathbf{v} \sqrt{m / K}$, and $\mathbf{a}_{n}=\mathbf{a} m S / K$. Normalized time is $t_{n}=t(1 / S) \sqrt{K / m}$, and the normalized magnetic field is $\mathbf{B}_{n}=\mathbf{B} q S / \sqrt{m K}$. Solving for the un-normalized parameters and substituting into Newton's second law, $m \mathbf{a}=q \mathbf{v} \times \mathbf{B}$, yields a normalized version of Newton's second law, $\mathbf{a}_{n}=\mathbf{v}_{n} \times \mathbf{B}_{n}$. The relation $B_{m}=\sqrt{2 m K /\left(q^{2} r_{m}^{2}\right)}$ is used to define a positive parameter $r_{m}$. Such a relation would be satisfied if $r_{m}$ equals the cyclotron radius of a particle that is located within a uniform magnetic field of magnitude $B_{m}$ and that has a kinetic energy $K$ associated with the cyclotron motion. Substitution into Eq. (3) and conversion to normalized parameters yields

$\mathbf{B}_{n}\left(x_{n}, y_{n}, z_{n}\right)=\operatorname{sgn}(q) \sqrt{2} r_{n m}^{-1} \beta\left(z_{n}, x_{n}\right)$,

where $\operatorname{sgn}(q)=q /|q|$ and $r_{n m}=r_{m} / S$.

The normalized equations of motion are

$$
\begin{aligned}
& x_{n}{ }^{\prime \prime}\left(t_{n}\right)=\operatorname{sgn}(q) \sqrt{2} r_{n m}^{-1} y_{n}^{\prime}\left(t_{n}\right) \beta_{z}\left[z_{n}\left(t_{n}\right), x_{n}\left(t_{n}\right)\right], \\
& y_{n}{ }^{\prime \prime}\left(t_{n}\right)=\operatorname{sgn}(q) \sqrt{2} r_{n m}^{-1}\left(z_{n}^{\prime}\left(t_{n}\right) \beta_{x}\left[z_{n}\left(t_{n}\right), x_{n}\left(t_{n}\right)\right]\right. \\
& \left.-x_{n}{ }^{\prime}\left(t_{n}\right) \beta_{z}\left[z_{n}\left(t_{n}\right), x_{n}\left(t_{n}\right)\right]\right),
\end{aligned}
$$


and

$z_{n}^{\prime \prime}\left(t_{n}\right)=-\operatorname{sgn}(q) \sqrt{2} r_{n m}^{-1} y_{n}{ }^{\prime}\left(t_{n}\right) \beta_{x}\left[z_{n}\left(t_{n}\right), x_{n}\left(t_{n}\right)\right]$.

Equations (6) to (8) were solved numerically to simulate single-particle trajectories. A measure of the inaccuracy of a simulated trajectory is the change of a particle's normalized kinetic energy, a conserved quantity. The normalized kinetic energy changed typically by less than $0.001 \%$ for each simulated trajectory reported here.

Plots of 1000 simulated classical trajectories are shown in Fig. (6) using the parameters for the magnetic field shown in Fig. (5). Each particle's trajectory starts at normalized time $t_{n}=0$ and at normalized spatial coordinates

$x_{n}(0)=x_{n 0}=R_{x}$,

$y_{n}(0)=y_{n 0}=0$,

$z_{n}(0)=z_{n 0}=5$.

Here, $R_{x}$ denotes a random number that is equally likely to have any value between 0 and 1 . Thus, the initial coordinate $x_{n 0}$ has a value that is randomly sampled over a distance equal to one spatial period of the periodic field. Such sampling of $x_{n 0}$ values is consistent with considering the spatial period of the field to be much smaller than the spatial variation of the source of incident charged particles. The configuration is treated as infinite in the $y$ dimension, and the value used for $y_{n 0}$ is unimportant. The value of $z_{n 0}$ is large enough for the effect of the magnetic field to be negligible near the start of each trajectory.

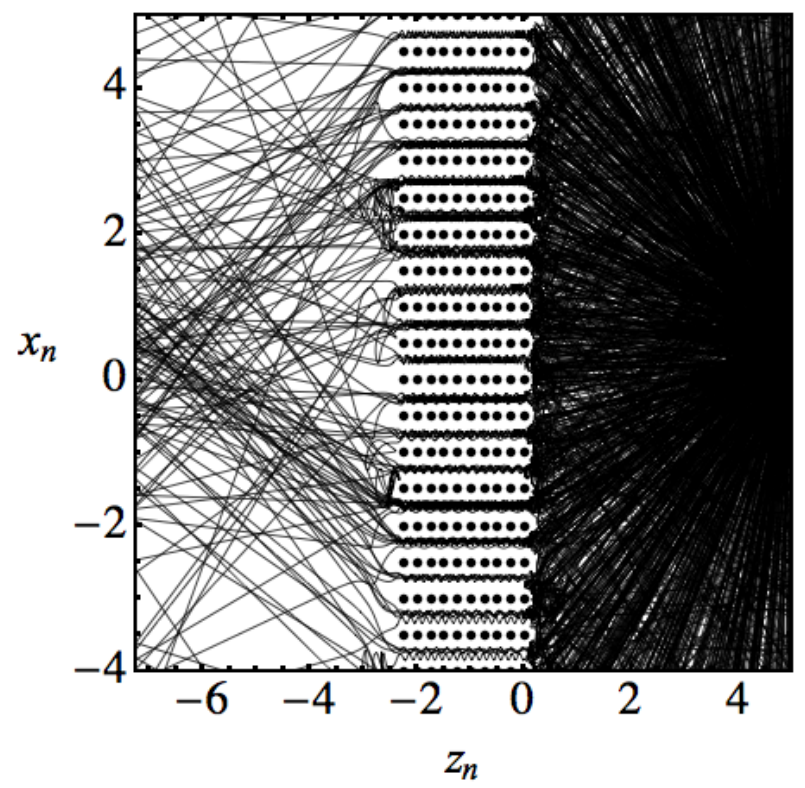

Fig. (6). Parametric plots of 1000 trajectories simulated using the parameters for the magnetic field shown in Fig. (5) and with $r_{n m}=0.05$. The dots indicate wire locations. See the text for details.

Let $\left(v_{n 0}, \theta_{0}, \varphi_{0}\right)$ denote spherical coordinates in velocity space associated with a particle's initial normalized velocity $\mathbf{v}_{n}(0)=\mathbf{v}_{n 0}$. The particle's initial Cartesian velocity components are

$v_{n x}(0)=v_{n x 0}=\sqrt{2} \sin \theta_{0} \cos \varphi_{0}$,

$v_{n y}(0)=v_{n y 0}=\sqrt{2} \sin \theta_{0} \sin \varphi_{0}$,

$v_{n z}(0)=v_{n z 0}=\sqrt{2} \cos \theta_{0}$,

where $v_{n 0}=\sqrt{2}$ is a particle's initial normalized speed (because $\left.K_{n 0}=1=12 v_{n 0}^{2}\right)$. $\theta_{0}$ must have a value in the range $\pi / 2<\theta_{0} \leq \pi$ for the particle to move toward the $z=0$ plane, and $\varphi_{0}$ has a value in the range $0 \leq \varphi<2 \pi$. The particles were considered to have a monoenergetic isotropic velocity distribution initially, except that particles that would initially travel away from the wires in the $\hat{\mathbf{k}}$ direction were excluded. Sampling expressions for the initial velocity-space angles are

$\theta_{0}=\arccos \left(-R_{\theta}\right)$,

$\varphi_{0}=2 \pi R_{\varphi}$.

Here, $R_{\theta}$ and $R_{\varphi}$ denote random numbers, and each is equally likely to have any value between 0 and 1 . The sampling expression for $\theta_{0}$ is derived by considering that the probability density function in spherical coordinates for a monoenergetic isotropic distribution of velocities is separable in the three variables. The $\theta_{0}$ probability density is $f_{\theta}\left(\theta_{0}\right)=\sin \theta_{0}$ for $\theta_{0}$ limited to values in the range $\pi / 2<\theta_{0} \leq \pi$. Then, $R_{\theta}=\int_{\pi / 2}^{\theta_{0}} f_{\theta}(\theta) d \theta$ is used to obtain the sampling expression for $\theta_{0}$.

Each magnetic cusp has a region within which the magnetic field has approximately straight field lines within the range $-2.07 \leq z_{n} \leq-0.18$. The effect of the magnetic field is negligible for $z_{n} \tilde{<}-7.25$ and $z_{n} \tilde{>} 5$ for the parameters considered. Each trajectory simulation was discontinued either when a particle reached $z_{n}=-8.25$ (after traversing the magnetic field) or when a particle reaches $z_{n}=6$ (after being reflected by the magnetic field). For the 1000 simulated trajectories shown in Fig. (6), a value $r_{n m}=0.05$ was chosen. The sign of $I_{1}$ is chosen to be the same as the sign of charge, $\operatorname{sgn}\left(I_{1}\right)=\operatorname{sgn}(q)$. However, statistically averaged Monte Carlo results are independent of $\operatorname{sgn}\left(I_{1}\right)$ and $\operatorname{sgn}(q)$, because $x_{n 0}$ is randomly sampled over one spatial period. (Simulations similar to those shown in Fig. (6) were run with all possible combinations of the values of $\operatorname{sgn}\left(I_{1}\right)$ and $\operatorname{sgn}(q)$, and similar results were obtained.)

\subsection{Fraction of Particles That Enter the Cusps}

The fraction $F_{v}$ of particles that enter the cusps and reach $z_{n}=-0.5$ has been evaluated using the same parameters as 
used for the simulations shown in Fig. (6), except that various values of $r_{n m}$ have been used. The number $N_{s}$ of simulated trajectories used was 10,000 for each value of $r_{n m}$. In Fig. (7), the fraction of particles that reached $z_{n}=-0.5$ is plotted versus $r_{n m}$. The solid line is a linear fit given by $F_{v}=3.4 r_{n m}$. The linear dependence found for the fit agrees with what may be expected according to prior theory [30]. When written in terms of unnormalized parameters, the fit is

$F_{v}=3.4 m v\left(|q| B_{m} S\right)^{-1}$.

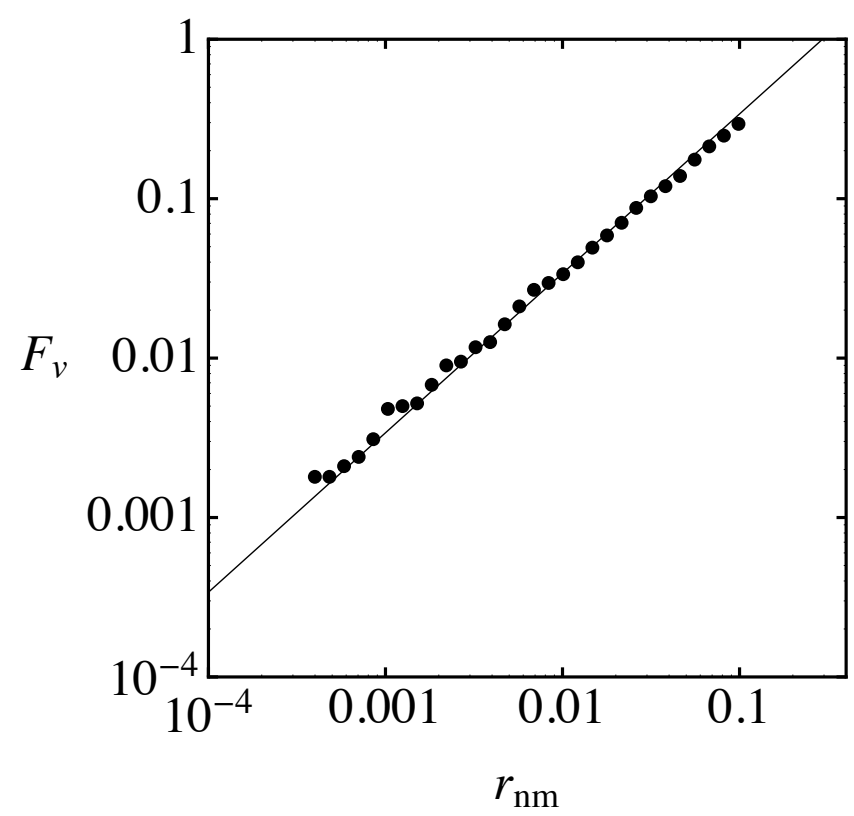

Fig. (7). Fraction of trajectories that reach $z_{n}=-0.5$, evaluated using the parameters for the magnetic field shown in Fig. (5). Also shown is a fit given by Eq. (12).

\subsection{Density Profile For Particles That Enter the Cusps}

The position distribution in the $x$ dimension for particles that enter the cusps and reach $z_{n}=-0.5$ has been evaluated using the same parameters as used for the simulations shown in Fig. (6). The number of simulated trajectories used was $N_{s}=100,000$. Each $x_{n}$ value at the time a trajectory reaches $z_{n}=-0.5$ was shifted to a corresponding location in the range $0<x_{n}<S_{n} / 2=0.5$. The range $0<x_{n}<S_{n} / 2$ was divided into $N_{b}=101$ bins of equal length. The number $N_{k}$ of trajectories that ended within the range of a bin labeled $k$ was determined for each bin. In Fig. (8), the values $N_{k}$ are plotted versus $x_{n k}$, which denotes the midpoint location of a bin. Also shown is a triangular fit having the form

$N_{v}=N_{m}\left(1-2\left|x_{n}-x_{n m}\right| w_{n}^{-1}\right) \Theta\left(1-2\left|x_{n}-x_{n m}\right| w_{n}^{-1}\right)$.

The values $x_{n m}=S_{n} / 4=0.25$ and $w_{n}=4 r_{n m}=0.2$ are used for the plot in Fig. (8). Here, $x_{n m}$ is the location where the position distribution reaches a maximum value $N_{m} ; w_{n}$ is the normalized full width at the base of the distribution; $\Theta$ is the Heaviside step function, which equals unity when its argument is positive and equals zero when its argument is negative. Equation (13) with $x_{n m}=S_{n} / 4$ and $w_{n}=4 r_{n m}$ has also been found to provide fits similar to the one shown in Fig. (8) for $r_{n m}$ values of $0.01,0.03,0.07$, and 0.09 .

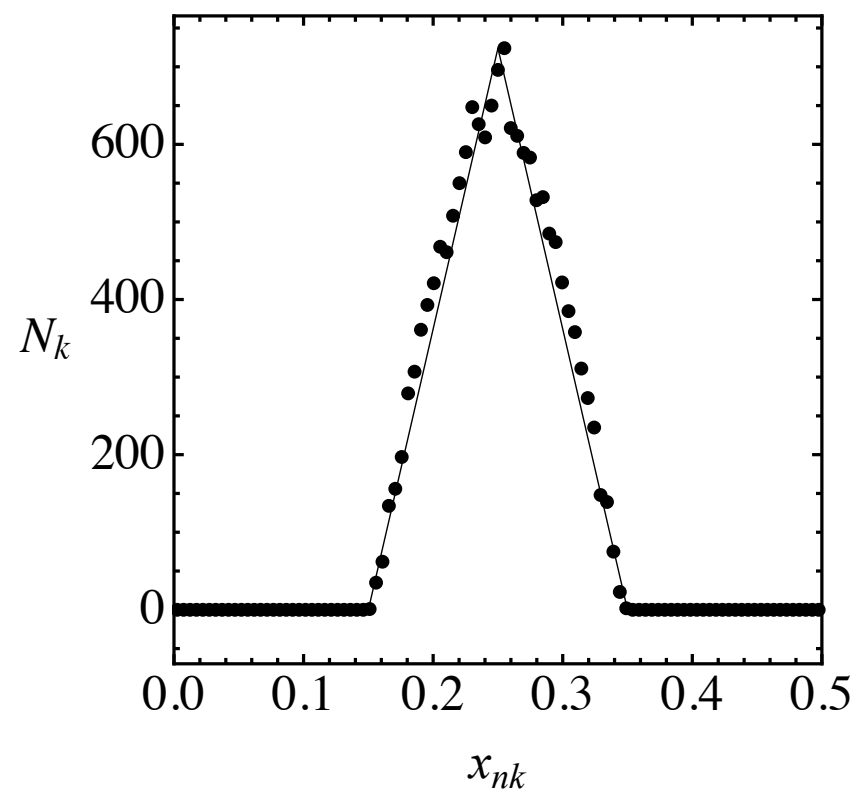

Fig. (8). Position distribution for particles at $z_{n}=-0.5$, evaluated using the parameters for the magnetic field shown in Fig. (5). Also shown is a fit given by Eq. (13).

The normalized width of a bin is $\Delta x_{n}=\left(S_{n} / 2\right) / N_{b}=0.5 / 101$. All $N_{s}=100,000$ trajectories started at $z_{n}(0)=5$ with randomly selected $x_{n}$ values within the range $0<x_{n}<S_{n}=1$. The average number of particles that started within the width of a bin was $N_{s k}=N_{s} \Delta x_{n} / S_{n}=495.05$. All particles moved at the same speed $v$. The density $n_{v}$ of particles within the straight field-line region of a cusp is taken to be proportional to $N_{v}$ given by Eq. (13). A relationship between the maximum particle density $n_{m}$ and the particle density $n_{0}$ outside the magnetic field (e.g., at $z_{n}=5$ ) is taken to be $n_{m} /$ $n_{0}=N_{m} / N_{s k}$, where $N_{m}$ is the number of trajectories that end within the range of a bin that has a midpoint located at $x_{n m}$. The value $n_{m} / n_{0}=1.38$ was found numerically to occur (to within $5 \%$ ) for $r_{n m}$ values of $0.01,0.03,0.07$, and 0.09 . The density $n_{v}$ of particles within the straight field-line region of a cusp (while traveling with $v_{z}<0$ ) is written in terms of unnormalized parameters as

$$
\begin{aligned}
& n_{v}(x)=1.38 n_{0}\left(1-\left|q\left(x-x_{m}\right)\right| B_{m}[2 m v]^{-1}\right) \\
& \times \Theta\left(1-\left|q\left(x-x_{m}\right)\right| B_{m}[2 m v]^{-1}\right),
\end{aligned}
$$

where $w=4 r_{m}=4 m v /\left(|q| B_{m}\right)$ has been used for the normalized full width at the base of the profile, and $x_{m}$ is the location where the density reaches a maximum value. 


\section{MAXWELLIAN VELOCITY DISTRIBUTION}

\subsection{Fraction of Incident Particles That Enter the Cusps}

The fraction of particles that enter the cusps and reach the straight field-line regions is now evaluated, considering the incident charged particles to have a non-drifting isotropic Maxwellian velocity distribution. The average particle speed associated with a non-drifting isotropic Maxwellian velocity distribution is $\langle v\rangle=\sqrt{8 T /(\pi m)}$, where $T$ is temperature in energy units. The replacement $v \rightarrow\langle v\rangle$ in Eq. (12) is the same as averaging Eq. (12) over a speed distribution. Such a replacement provides the fraction of particles with a nondrifting isotropic Maxwellian velocity distribution that reach the straight field-line region of a cusp:

$F=6.8\left(|q| B_{m} S\right)^{-1} \sqrt{2 m T \pi^{-1}}=5.4 r S^{-1}$.

Here, $r=\sqrt{m T} /\left(|q| B_{m}\right)$ is the cyclotron radius scale length for a particle that is located within a magnetic field of strength $B_{m}$.

\subsection{Density Profile For Particles That Enter the Cusps}

The density profile for particles located within the straight field-line region of a cusp and that travel with $v_{z}<0$ is now evaluated for incident particles that have a non-drifting isotropic Maxwellian velocity distribution. The density profile is evaluated by integrating Eq. (14) over the speed probability density,

$f_{v}(v)=(4 / \sqrt{\pi})(m /[2 T])^{3 / 2} v^{2} e^{-m v^{2} /(2 T)}$.

The result is

$$
\begin{aligned}
& n(x)=1.38 n_{0} \int_{0}^{\infty}(1-u / v) \Theta(v-u) f_{v}(v) d v \\
& =1.38 n_{0} \operatorname{erfc}\left(\left|x-x_{m}\right| /[\sqrt{8} r]\right),
\end{aligned}
$$

where $u=\left(\left|q\left(x-x_{m}\right)\right| B_{m} /(2 m)\right.$, and erfc is the complementary error function. A few sample calculations illustrate how rapidly the density decreases away from $x=x_{m}: \quad n / n_{0}=8 \times 10^{-7} \quad$ for $\quad\left|x-x_{m}\right| / r=10$; $n / n_{0}=2 \times 10^{-23}$ for $\left|x-x_{m}\right| / r=20$; and $n / n_{0}=1 \times 10^{-50}$ for $\left|x-x_{m}\right| / r=30$.

\section{ELECTROSTATIC PLUGGING SIMILAR TO THAT WITHIN A NESTED PENNING TRAP}

As indicated by Fig. (5), each cusp has a region within which the magnetic field has approximately straight magnetic field lines. The magnetic field is uniform within a region of straight field lines for noncurved line cusps. According to Eq. (17), plasma particles that enter the cusps (in the single-particle limit considered in the present work) will enter a uniform magnetic field region, provided that the cyclotron radius scale length $r$ has a sufficiently small value. In such a case, it is possible to consider the particle motion within the guiding center approximation, whereby a particle's position is averaged over its cyclotron motion. The conditions are similar within nested Penning traps, and the knowledge base developed regarding plasma confinement within nested Penning traps provides guidance for considering the present concept.

An electric field can be used to reflect a charged particle that travels parallel to a uniform magnetic field. For plasma particles associated with temperature $T$ in energy units, a condition for good confinement by an electric field within a Penning trap is $\Delta U \gg T$, where $\Delta U$ is the total increase in potential energy that a particle would experience in passing all the way through the self-consistently produced electric field. For reflecting a charged particle regardless of its charge sign, two consecutive regions that contain oppositely directed electric fields can be used. The change in potential associated with the second electric field region must be sufficiently large to reflect particles that are necessarily accelerated through the first electric field region.

Three different plasma equilibria may be possible within a nested Penning trap, such that a plasma with an electrically neutral overlap region is confined [4-7]. In one equilibrium, two plasma species have different temperatures and all particles have equal charge magnitudes. The temperature (in energy units) of the plasma species confined within an inner well (see Fig. 1) must be much smaller than the depth of the potential (energy) well that confines it. The temperature of the plasma species confined within a nested (and inverted) outer well must be either about the same or larger than the depth of the inner well for the outer-species plasma to overlap the confinement volume of the inner-species plasma. In a second equilibrium, the two species have the same temperature, and the particles of each species have disparate charge states. For the third equilibrium, the two species have the same temperature and all particles have equal charge magnitudes, but the plasma species confined by the outer well has a non-Maxwellian velocity distribution outside of the inner well. More details about possible plasma equilibria within nested Penning traps may be found in Refs. [4-7].

There exist a variety of multiple-particle effects (including collective effects) that have been found to be important within nested Penning traps [3-7]. Such effects, which may also be important for the present concept, are beyond the scope of the single-particle limit considered here, and they will be considered in more detail in future work. Only a brief discussion of multiple-particle effects is given here. Collisions among particles can affect the first and third equilbria described within the preceding paragraph. Collisions tend to cause the two species in the first equilibrium to thermalize eventually and reside in separated confinement volumes or to be lost from confinement. Collisions tend to cause particles from one species in the third equilibrium to become trapped outside of the inner well. The two-stream instability could have a similar effect if the size of the non-Maxwellian plasma region located outside of the inner well is too large. Non-neutral regions of plasma can affect all three equilibria. For example, the plasma located outside of the inner well is not neutral, and the plasma density can be limited by space charge effects. The self-consistently produced electric field can have a component that is perpendicular to the magnetic field and cause plasma particles to experience an $\mathbf{E} \times \mathbf{B}$ drift. Because 
noncurved line cusps are necessarily of finite extent, direct particle losses due to an $\mathbf{E} \times \mathbf{B}$ drift may occur. However, such losses can be avoided, in principal, if only ring cusps and point cusps are used to produce a force field.

Suppose that one of the three equilibria mentioned above is established using the configuration illustrated in Fig. (3d), and that the plasma is electrically neutral and effectively unmagnetized near the geometric center of the trap. There exist effects that can cause non-neutral regions to form. If the loss rate of one species is larger than the other, then a non-neutral plasma sheath may form along the periphery of the unmagnetized plasma. It is hypothesized that separate control of the electric confinement of each species by using applied electrostatic voltage variations similar to those found in nested Penning traps may allow a confinement equilibrium that is devoid of a non-neutral plasma sheath. Collisions will tend to cause electrons to diffuse into the magnetic field at a faster rate than ions, and the effect can cause the formation of a non-neutral layer with negative space charge within the magnetic field. On the other hand, Eq. (17) predicts a mass-dependent width for the density profile of a particle species that enters a cusp. For example, for an electron-ion plasma, with both species having the same temperature, the width of the ion density profile associated with ions that enter a cusp will be larger than that of the electron density profile. Such an effect can form a non-neutral layer with positive space charge within the magnetic field. It is hypothesized that an equilibrium is possible, whereby electrons that diffuse into the magnetic field distribute themselves so as to neutralize the ion space charge within the magnetic field. The hypothesized equilibria mentioned here will be sought in future work.

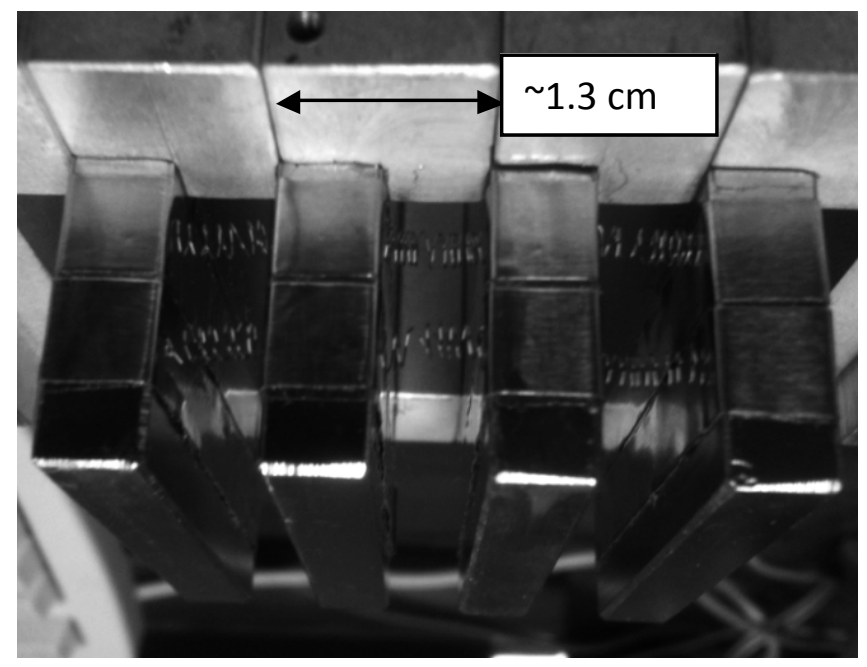

Fig. (9). Experimental force field segment. (Compare to Fig. 3b). The four permanent magnets can be seen protruding from the clamp assembly. Electrostatic plugging is provided by copper electrodes that wrap around each magnet.

\section{EXPERIMENT}

As an initial attempt to develop a force field, a few periods of such have been created and experimentally tested [37]. The experimental setup is shown in Fig. (9). Four neodymium-iron-boron permanent magnets (dimensions: $5.08 \mathrm{~cm} \times 5.08 \mathrm{~cm} \times 0.635 \mathrm{~cm}, \sim 1 \mathrm{~T}$ maximum field) were clamped with like poles facing each other and with a separation of $0.64 \mathrm{~cm}$ in between them. Copper electrodes were attached to, but were electrically isolated from, the magnets in order to achieve electrostatic plugging of the magnetic cusps. To perform the initial experimental testing, the magnet structure was placed in a vacuum chamber where an Ar plasma was generated using a DC glow discharge. The plasma source consisted of a straight tungsten wire encircled by a tungsten wire loop, with a voltage difference applied between them to create a discharge. To obtain charged particles from the discharge region, an einzel-lens-like configuration of three electrodes was employed, with grounded first and last electrodes and a biased middle electrode. These extraction electrodes produced an electric field that penetrated the discharge region and extracted a species of a particular sign of charge. Several conditions (background pressure, discharge voltage, source bias with respect to ground, extraction electrode voltage) were empirically optimized to obtain a visible plasma. It was found that, for the present system, a background pressure of 120 mTorr and a discharge voltage of $\sim 200 \mathrm{~V}$ were sufficient to sustain a plasma that could be imaged. Extraction was achieved with the additional condition that the tungsten wires were biased to $\sim 80 \mathrm{~V}$ with respect to the established ground, and the extraction electrode voltage was held at $-660 \mathrm{~V}$. Extraction of electrons from the discharge region was achieved by literally reversing all the voltages but increasing the discharge voltage to $\sim 300 \mathrm{~V}$ to keep the discharge stable. Ar ions (or electrons) were extracted from the discharge region and directed onto the magnet assembly. These Ar ions (or electrons) diffused through a region where the background pressure was relatively high (120 mTorr), with a mean free path of $\sim 0.05 \mathrm{~cm}$. The $\mathrm{Ar}$ ions (or electrons) collisionally excited residual gas atoms, which primarily consisted of Ar atoms. An ST-7 SBIG CCD camera was employed to record light emitted by deexcitations, using typical integration times of 2 or 3 minutes. The plasma was imaged with electrostatic plugging either turned off or turned on. Some resulting images are presented in Fig. (10) for ion extraction and Fig. (11) for electron extraction.

\section{CONCLUSION}

In conclusion, research has been reported on the properties of a short-range, static, electromagnetic force field that appears to be capable of reflecting incident charged particles of either sign of charge at any angle of incidence. Because of the wide variety of uses of plasmas, and the increasing uses of antimatter plasmas, basic studies of force fields may lead to a variety of applications. For example, force fields might be useful for confining antimatter or fusion plasmas, or for plasma thrusters or ion sources. A force field would merge two concepts that have been studied recently: the nested Penning trap and the artificially structured boundary. A nested Penning trap provides simultaneous confinement of oppositely signed plasma species by applying nested electrostatic potential wells along a magnetic field. An artificially structured boundary produces a spatially periodic static field, such that the spatial period and range of the field is much smaller than the 

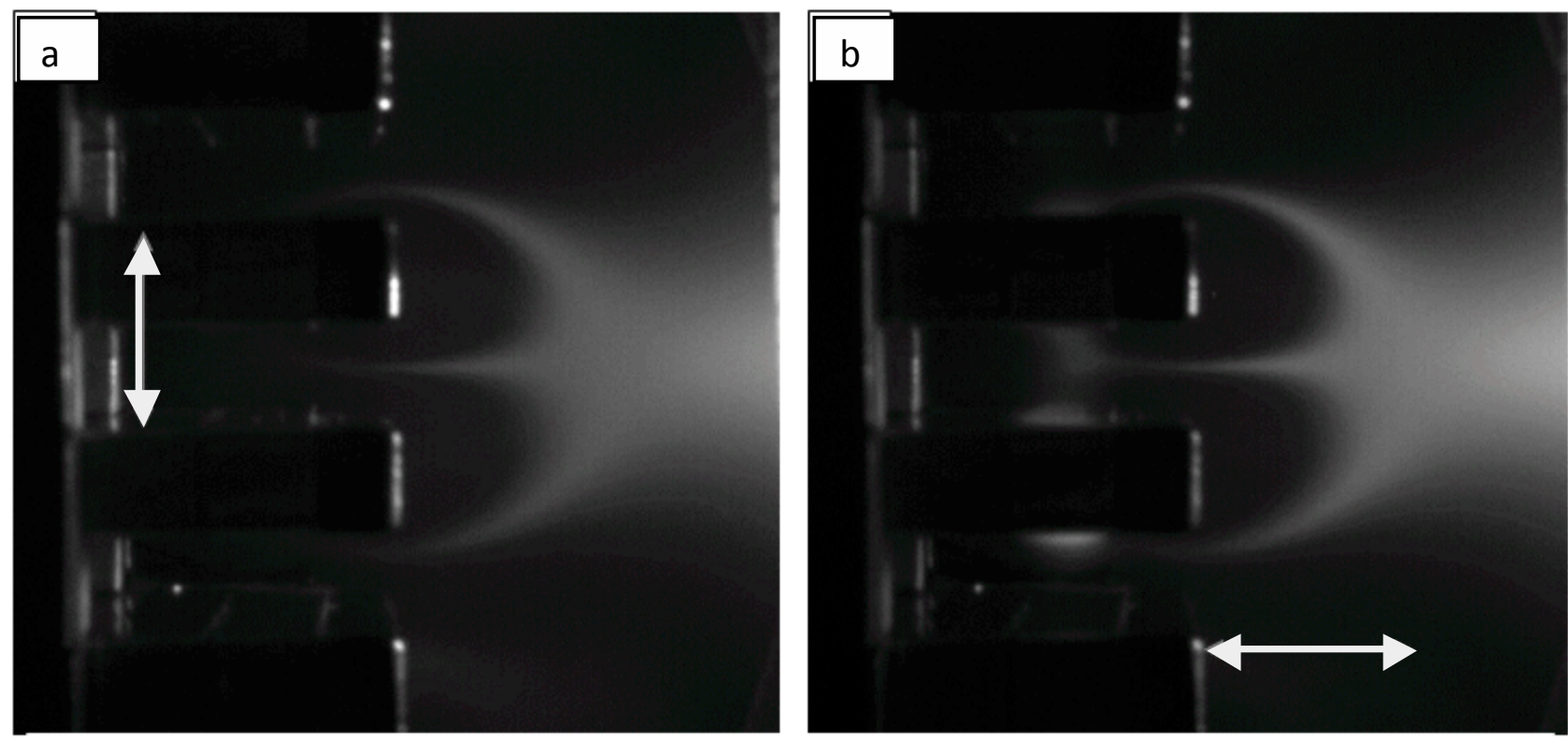

Fig. (10). (a) Ar ions incident on magnet structure with electrostatic plugging turned off (electrodes, magnets, and supporting structure at ground voltage). (b) Electrostatic plugging turned on (reflection electrodes at $\sim 30 \mathrm{~V}$ ). Arrows represent a $\sim 1.3 \mathrm{~cm}$ length. Positively charged particles enter magnetic cusps in both (a) and (b). In (b) particles that enter a cusp experience an $\mathbf{E} \times \mathbf{B}$ drift that guides them into, or out of, the plane of the page, thereby extending the plasma perpendicular to the plane of the page. The brightness is enhanced where the $\mathbf{E} \times \mathbf{B}$ drifts occur.
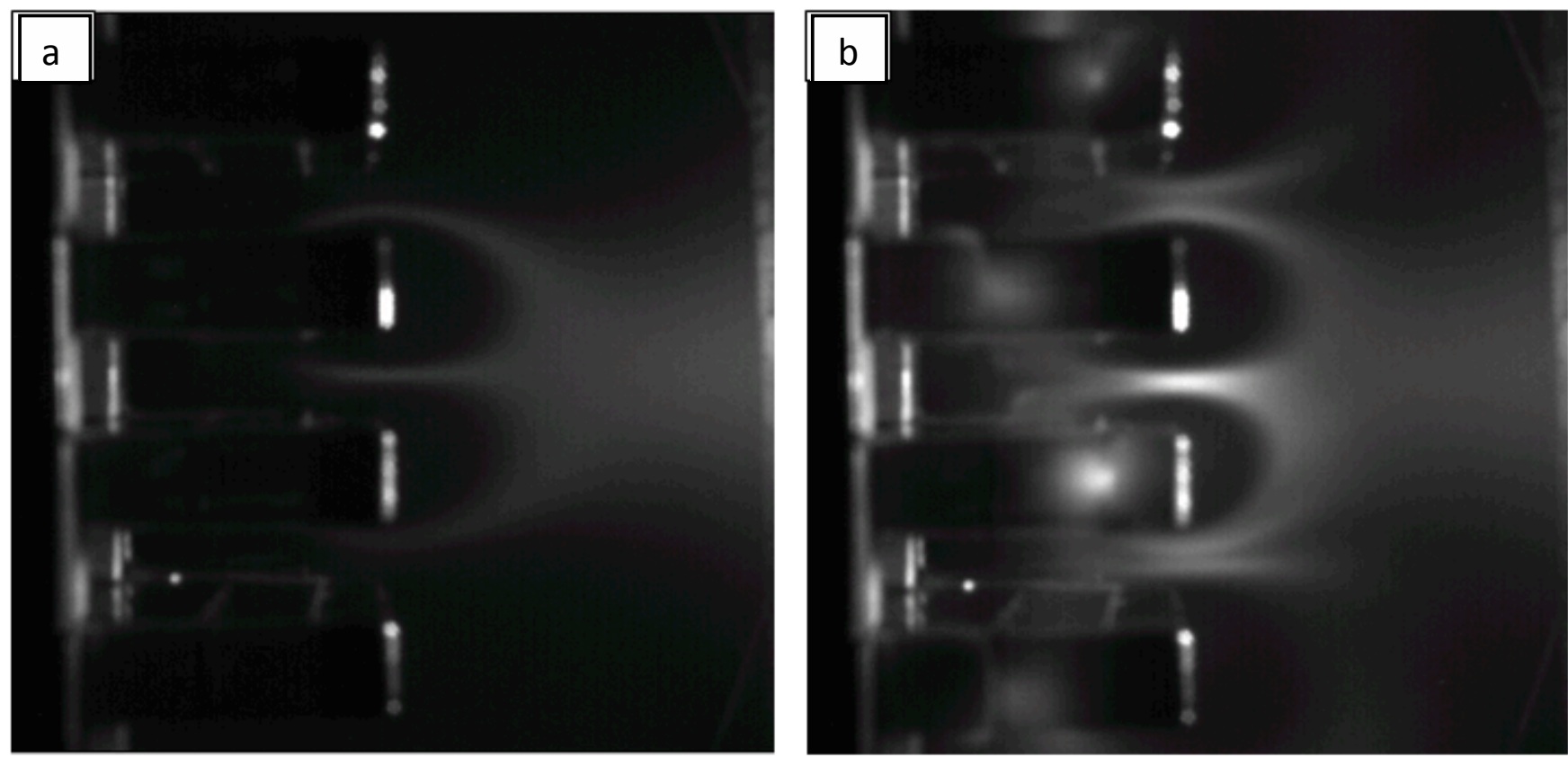

Fig. (11). Electrons incident on magnet structure (a) with electrostatic plugging turned off, and (b) with electrostatic plugging ( -200 V) turned on (b). In (b) the $\mathbf{E} \times \mathbf{B}$ drift caused the plasma to reach and pass in front of the ends of the magnets closest to the camera.

dimensions of a plasma that is confined by the field. A force field is envisioned as consisting of a spatially periodic sequence of magnetic cusps that are electrostatically plugged using applied electrostatic voltage variations similar to those found in nested Penning traps.

\section{ACKNOWLEDGMENTS}

This material is based upon work supported by the Department of Energy under Grant No. DE-FG0206ER54883. An earlier version of this paper was presented at the 47th AIAA/ASME/SAE/ASEE Joint Propulsion Conference \& Exhibit, Paper No. AIAA 2011-6120.

\section{CONFLICT OF INTEREST}

Declared none.

\section{REFERENCES}

[1] Dubin DHE, O'Neil TM. Trapped nonneutral plasmas, liquids, and crystals (the thermal equilibrium states). Rev Mod Phys 1999; 71: $87-172$. 
[2] Gabrielse G, Rolston SL, Haarsma L, Kells W. Antihydrogen production using trapped plasmas. Phys Lett A 1988; 129: 38-42.

[3] Ordonez CA, Weathers DL. Two-species mixing in a nested Penning trap for antihydrogen trapping. Phys Plasmas 2008; 15: 083504 .

[4] Ordonez CA. Confinement of a neutral plasma using nested electric potential wells. Phys Plasmas 1997; 4: 2313-5.

[5] Ordonez CA, Dolliver DD, Chang Y, Correa JR. Possibilities for achieving antihydrogen recombination and trapping using a nested Penning trap and a magnetic well. Phys Plasmas 2002; 9: 3289302.

[6] Dolliver DD, Ordonez CA. Confinement physics for thermal, neutral, high-charge-state plasmas in nested-well solenoidal traps. Phys Rev E 1999; 59: 7121-7.

[7] Ordonez CA. Time-dependent nested-well plasma trap. IEEE Trans Plasma Sci 1996; 24: 1378-82.

[8] Amoretti M, Amsler C, Bonomi G, et al. Production and detection of cold antihydrogen atoms. Nature 2002; 419: 456-9.

[9] Gabrielse G, Bowden NS, Oxley P, et al. Background-free observation of cold antihydrogen with field-ionization analysis of its states. Phys Rev Lett 2002; 89: 213401.

[10] Enomoto Y, Kuroda N, Michishio K, et al. Synthesis of cold antihydrogen in a cusp trap. Phys Rev Lett 2010; 105: 243401.

[11] Andresen GB, Ashkezari MD, Baquero-Ruiz M, et al. Trapped antihydrogen. Nature 2010; 468: 673-6.

[12] Andresen GB, Ashkezari MD, Baquero-Ruiz M, et al. Confinement of antihydrogen for 1,000 seconds. Nat Phys 2011; 7: 558-64.

[13] Robicheaux F. Atomic processes in antihydrogen experiments: a theoretical and computational perspective. J Phys B 2008; 41: 192001.

[14] Gabrielse G. Atoms made entirely of antimatter: two methods produce slow antihydrogen. Adv Atom Mol Opt Phys 2005; 50: 155-217.

[15] Fabris D, Belov AS, Bonomi G, et al. The AEGIS detection system for gravity measurements. Nucl Phys A 2010; 834: 751c-753c.

[16] Charlton M, Eades J, Horvath D, Hughes RJ, Zimmermann C. Antihydrogen physics. Phys Rep 1994; 241: 65-117.

[17] Holzscheiter MH, Charlton M, Nieto MM. The route to ultra-low energy antihydrogen. Phys Rep 2004; 402: 1-101.

[18] Surko CM, Greaves RG. Emerging science and technology of antimatter plasmas and trap-based beams. Phys Plasmas 2004; 11: 2333-48

[19] Madsen N. Cold antihydrogen: a new frontier in fundamental physics. Philos Trans R Soc A 2010; 368: 3671-82.

[20] Ordonez CA. Effect of positron space charge on operation of an antihydrogen trap. Phys Rev E 2007; 76: 017402.

[21] Gabrielse G, Kolthammer WS, McConnell R, et al. Adiabatic cooling of antiprotons. Phys Rev Lett 2011; 106: 073002.
[22] Andresen GB, Ashkezari MD, Baquero-Ruiz M, et al. Autoresonant excitation of antiproton plasmas. Phys Rev Lett 2011; 106: 025002 .

[23] Gabrielse G, Kolthammer WS, McConnell R, et al. Centrifugal separation of antiprotons and electrons. Phys Rev Lett 2010; 105: 213002.

[24] Andresen GB, Ashkezari MD, Baquero-Ruiz M, et al. Evaporative cooling of antiprotons to cryogenic temperatures. Phys Rev Lett 2010; 105: 013003.

[25] Ordonez CA. Drifting plasma confinement with a spatially periodic field. IEEE Trans Plasma Sci 2010; 38: 388-92.

[26] Ordonez CA. Charged particle reflection from an artificially structured boundary that produces a spatially periodic magnetostatic field. J Appl Phys 2009; 106: 024905.

[27] Ordonez CA. Effect of a periodic electrostatic potential on magnetized particle transport. Phys Plasmas 2008; 15: 114507.

[28] Ordonez CA. Charged particle transport through a periodic electrostatic potential having a small spatial period. J Appl Phys 2008; 104: 054903.

[29] Correa JR, Ordonez CA, Weathers DL. Transverse confinement of an ion beam in a purely electrostatic configuration. Nucl Instrum Methods Phys Res B 2005; 241: 909-12.

[30] Dolan TJ. Magnetic electrostatic plasma confinement. Plasma Phys Contr Fusion 1994; 36: 1539-94; and references therein

[31] Mohri A, Yuyama T, Kiwamoto Y, Yamazawa Y, Michishita T. Confinement of nonneutral plasmas in a trap composed of a cusped magnetic field and an electrostatic octapole field. Jpn J Appl Phys 1998; 37: L1553-L1555.

[32] Mohri A, Yamazaki Y. A possible new scheme to synthesize antihydrogen and to prepare a polarised antihydrogen beam. Europhys Lett 2003; 63: 207-13.

[33] Saitoh H, Mohri A, Enomoto Y, Kanai Y, Yamazaki Y. Radial compression of a non-neutral plasma in a cusp trap for antihydrogen synthesis. Phys Rev A 2008; 77: 051403.

[34] Lavrent'ev OA, Maslov VA, Germanova SV, Nozdrachov MG, Oboznyj VP, Shevchuk BA. Modeling of a starting mode of thermonuclear reactor "Elemag." Fusion Sci Technol 2005; 47: 224-7.

[35] Dey I, Bhattacharjee S. Penetration and screening of perpendicularly launched electromagnetic waves through bounded supercritical plasma confined in multicusp magnetic field. Phys Plasmas 2011; 18: 022101; and references therein.

[36] Martinez-Sanchez M, Ahedo E. Magnetic mirror effects on a collisionless plasma in a convergent geometry. Phys Plasmas 2011; 18: 033509; and references therein.

[37] Pacheco JL, Ordonez CA, Weathers DL. Plasma interaction with a static spatially periodic electromagnetic field. IEEE Trans Plasma Sci 2011; 39: 2424-5. 